\title{
Remote Detection Sodium Cell Magnetometry using Amplitude Modulated Light
}

\author{
Renu Tripathi ${ }^{1, *}$, Christiane Ebongue ${ }^{1}$, Lorna Caesar ${ }^{1}$, Gour Pati ${ }^{1}$, Anthony Yu$^{2}$, Michael Krainak ${ }^{2}$ \\ ${ }^{1}$ Dept. of Physics and Engineering, Delaware State University, Dover, DE 19901, USA \\ Laser \& Electro-Optics Branch, NASA GSFC, Greenbelt, MD 20771, USA \\ "rtripathi@desu.edu
}

\begin{abstract}
We have investigated the performance of a remote-detection sodium cell magnetometer using synchronous optical pumping with amplitude modulated light. The magnetometer is used to measure ambient geomagnetic field with high-sensitivity using backscattered resonance fluorescence.

OCIS codes: (270.1670) Coherent optical effects; (020.3690) Line shapes and shifts; (300.6320) Spectroscopy, highresolution; (120.3930) Metrological instrumentation.
\end{abstract}

\section{Introduction}

Laser Guide Star (LGS) used in adaptive optical astronomy takes advantage of laser excitation in the atomic sodium layer in the mesosphere. The technology can be leveraged in designing ground-based magnetometer for measuring geomagnetic field around $90 \mathrm{~km}$ above the earth's surface [1]. This relies on using an amplitude-modulated LGS probe laser for exciting sodium atoms, and measuring backscattered fluorescence from the sodium layer at the ground station. Synchronous optical pumping of sodium atoms with an amplitude-modulated probe laser can produce atomic spin alignment and an increase in backscattered light when the modulation frequency of the laser is matched to the Larmor frequency, $\mathrm{f}_{\mathrm{L}}=\gamma \mathrm{B}$, where $\gamma=7 \mathrm{~Hz} / \mathrm{nT}$ is the gyromagnetic ratio of $\mathrm{Na}$ atoms [2]. A magnetic resonance in backscattered fluorescence is produced by sweeping the modulation frequency of the laser around the Larmor frequency. Remote measurement of geomagnetic field can find many potential applications such as in large-scale mapping of magnetic structures, detection of oceanic currents, and the study of electric-current fluctuations in the ionosphere. A sensitive remote magnetometer capable of operating over a large distance (hundreds of meters) can also find challenging military applications such as magnetic-anomaly detection which involves ordnance detection and mine reconnaissance etc.

In this study, we demonstrate remote magnetic field measurements using a sodium cell by emulating the experimental method described above over a distance scale of few meters inside the laboratory. Sodium atoms in a vapor cell are interrogated with an amplitude-modulated light produced by an acousto-optic modulator (AOM), introduced in the beam path of a frequency-doubled amplified sodium laser. Magnetic resonance is detected in backscattered light, thus, allowing us to measure the magnetic field remotely. Characteristics of magnetic resonance such as linewidth and contrast (or SNR), and their dependence on optical power density, laser linewidth, B-field orientation and intensity are studied. Experimental measurements are also carried out to determine the sensitivity of the remote magnetometer operating in an unshielded environment.

\section{Experimental Description}

Fig.1a shows the experimental setup used in the remote magnetic field measurement. The light source used in our experiment is a frequency-doubled amplified diode laser producing narrow-band emission $(\Delta v<1 \mathrm{MHz})$ at $\lambda=$ $589.15 \mathrm{~nm}$ for resonant excitation of sodium atoms using the $\mathrm{D}_{2}$ manifold. The laser system consists of a low-power distributed feedback (DFB) laser (operating as the seed laser near $1178.3 \mathrm{~nm}$ ) whose output is fiber-coupled to a tapered amplifier (TA) chip to amplify the seed laser output to about $0.5 \mathrm{~W}$. Second harmonic generation in a long periodically-poled lithium niobate (PPLN) crystal converts $1178.3 \mathrm{~nm}$ to $589.15 \mathrm{~nm}$ for resonant excitation of sodium atoms in the vapor cell. The laser system parameters (e.g. TA current, laser mode, and crystal phase-matching temperature etc.) are optimized to produce maximum power $(\simeq 1 \mathrm{~mW})$ at $589.15 \mathrm{~nm}$ for our experiment. Fig. $1 \mathrm{~b}$ shows the picture of the laser system mounted on a breadboard for easy portability inside the laboratory.

The sodium cell assembly consists of a glass-heating coil unit wrapped around the cell and a three-axis Helmholtz coil with the sodium cell mounted at its center. The glass-heating coil uses recirculating silicon oil from a heated oil bath to heat the sodium cell up to a desired temperature, typically above the melting temperature $98^{\circ} \mathrm{C}$ of sodium. Such a heating does not produce ambient magnetic field in the cell environment, which is crucial for sensitive magnetic field measurements. The three-axis Helmholtz coil is used to cancel earth's magnetic field at the center of the coil where the cell is mounted, and, also, for applying magnetic field in an arbitrary direction with respect to light propagation in the cell. 


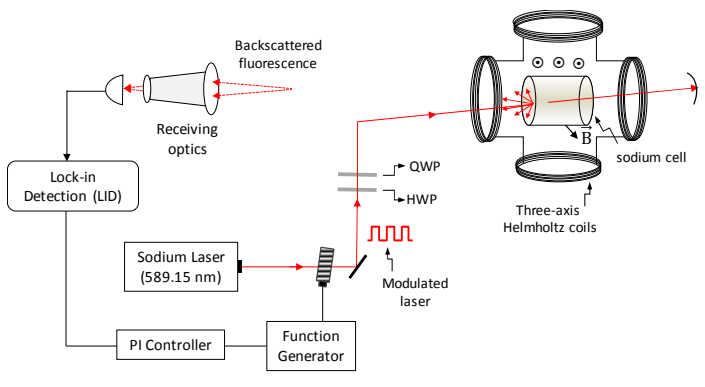

(a)

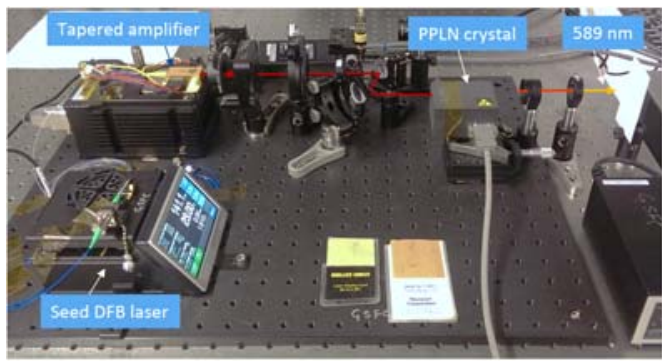

(b)

Figure 1 (a) Experimental setup developed using a sodium cell for remote magnetic field measurement, and (b) Breadboard mounted sodium laser system developed using nonlinear frequency-doubling of laser output from the amplified DFB laser.

\section{Results and Discussions}

Fig. 2a shows energy levels in the $\mathrm{D}_{2}$ manifold of sodium atoms. The ground state consists of two hyperfine states $\mathrm{F}=1$ and $\mathrm{F}=2$ which are separated by $1.771 \mathrm{GHz}$. Fig. $2 \mathrm{~b}$ shows the Doppler-broadened absorption spectrum of sodium atoms observed in transmitted light through the cell while scanning the laser wavelength around the $\mathrm{D}_{2}$ transitions. The absorption spectrum has two dips corresponding to the following transitions: a) $F=2 \rightarrow F^{\prime}(1,2,3)$, referred to as $D_{2 a}$ transition and b) $F=2 \rightarrow F^{\prime}(1,2)$, referred to as $D_{2 b}$ transition. The two dips are separated by 1.771 GHz. The width and depth of the absorption dips are affected by the cell temperature.

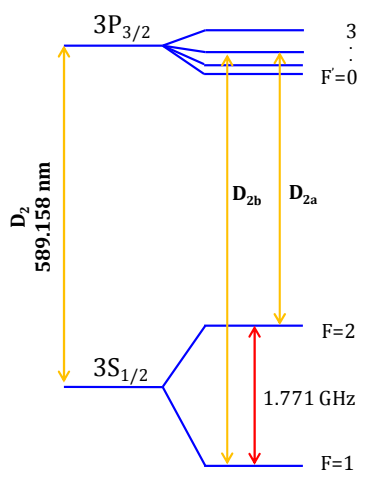

(a)

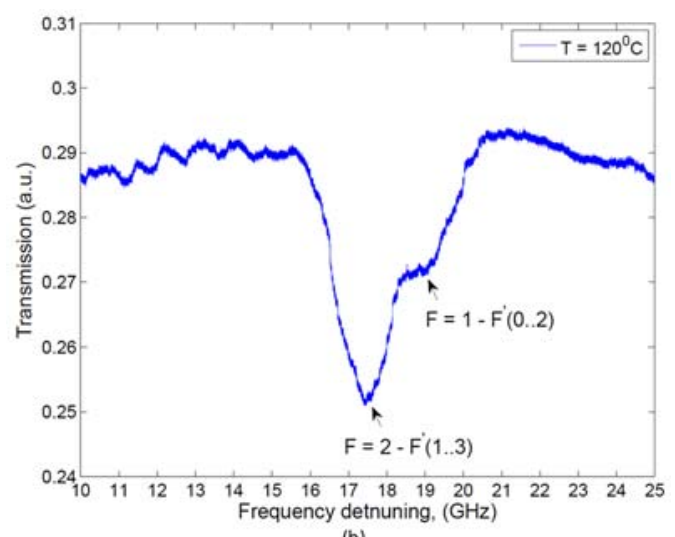

(b)

Figure 2 (a) Sodium energy levels in the $D_{2}$ manifold showing hyperfine ground and excited states, and (b) Doppler broadened $D_{2}$ line absorption spectrum observed in the transmitted light through the sodium cell.

For remote B-field measurement, the wavelength of the sodium laser is locked to the center $\mathrm{D}_{2 \mathrm{a}}$ absorption spectrum by using dichroic atomic vapor spectroscopy by sending a small fraction of the light through a reference sodium cell. Amplitude-modulated light is produced by passing the main laser beam through an AOM as shown in fig. 1a. A combination of half-wave and quarter-wave plates provides polarization control of the beam. Optical pumping of atoms is produced by circularly polarized light. Backscattered fluorescent light from the sodium cell is collected by a lens and focused onto the light-sensitive photodiode. Precautions have been taken to avoid backward reflections from various optical components from entering the receiving optics.

A function generator with a voltage-controlled oscillator (VCO) is used to drive the AOM to produce amplitude modulated light. Lock-in detection of the photodiode output is used to observe the magnetic resonance by sweeping the modulation frequency of the laser around the Larmor frequency. Remote magnetic field measurements are performed by locking the VCO to the peak of magnetic resonance using the electronic feedback from the proportionalintegral (PI) controller. The output voltage from the PI controller is converted to measure the applied magnetic field intensity. Power spectral density of the PI output voltage is obtained to measure the sensitivity of the remote magnetometer operating in the unshielded environment. The work is currently in progress and results will be forthcoming.

\section{References}

1. J. M. Higbie, S. M. Rochester, B. Patton, R. Holzlohner, D. Bonaccini Calia, and D. Budker, "Magnetometry with mesospheric sodium," Proc. Natl. Acad. Sci. 108(9), 3522-3525 (2011).

2. $\quad$ W. E. Bell and A. L. Bloom, "Optically driven spin precession," Phys. Rev. Lett. 6(6), 280-281 (1961). 